\title{
Chemical Proteomics: A Powerful Tool for Exploring Protein Lipidation
}

\author{
Elisabeth M. Storck ${ }^{1,2}$, Remigiusz A. Serwa ${ }^{1}$, Edward W. Tate ${ }^{1, *}$ \\ ${ }^{1}$ Department of Chemistry, Imperial College London, Exhibition Road, London, SW7 2AZ, UK \\ ${ }^{2}$ Centre for Pharmacology and Therapeutics, Department of Medicine, Imperial College London, \\ Du Cane Road, London, W12 0NN, UK
}

*Corresponding author: Dr. E.W. Tate, Department of Chemistry, Imperial College London, Exhibition Road, London, SW7 2AZ, UK; e.tate@imperial.ac.uk

\begin{abstract}
The study of post-translational modifications such as protein lipidation is a non-trivial challenge of the post-genomic era. In recent years the field of chemical proteomics has greatly advanced our ability to identify and quantify protein lipidation. In this mini-review we give a brief overview of the tools available to study protein acylation, prenylation and cholesterylation, and their application in the identification and quantification of protein lipidation in health and disease.
\end{abstract}

\begin{abstract}
Abbreviations: 17-ODYA, 17-octadecynoic acid; ABE, acyl-biotin exchange; acyl-RAC, resin assisted capture of S-acylated proteins; FTase, farnesyl transferase; FTI, farnesyl transferase inhibitor; Hh, Hedgehog protein; NMT, N-myristoyl transferase; PTM, post-translational modification; SILAC, stable isotope labelling with amino acids in cell culture
\end{abstract}

Key Words: Chemical proteomics, fatty acylation, prenylation, cholesterylation, SILAC

\section{Introduction}

Post-translational modifications (PTMs) of proteins include a host of chemical transformations that occur to proteins after (or occasionally during) ribosomal synthesis. A large number of reversible and irreversible PTMs serve to regulate protein function, activity and location within the cell. As PTMs are not directly encoded by the genome, they add a further layer of complexity to the proteome that is not readily accessible to the traditional tools of genetics. PTMs include the covalent attachment of small inorganic molecules (e.g. phosphorylation), carbohydrates (e.g. glycosylation), small proteins (e.g. ubiqutination) and lipids. Protein lipidation includes, among others, $N$-myristoylation [1], $S$ - and $N$-palmitoylation [2], prenylation [3], cholesterylation [4] and the attachment of glycosylphosphatidylinositol (GPI) anchors [5].

The importance of protein lipidation in normal and dysfunctional cell regulation is mirrored in current programs that aim to develop therapies targeting lipidation [6-8]. Modulating or inhibiting lipidation has an impact in a range of pathologies, including cancer, cardiovascular disease, fungal infections, tropical diseases such as malaria, as well as HIV and AIDS [1, 9, 10].

(FIGURE 1. Protein lipid modifications and a selection of chemical probes to detect protein lipidation. Sonic hedgehog (Shh) is N-palmitoylated on Cys25 and O-cholesterylated on Gly198. HRas is S-palmitoylated on Cys181/Cys184 and farnesylated on Cys186. Tyrosine protein kinase c-Src is $N$-myristoylated on Gly2. The insert boxes display a selection of azide/alkyne-tagged chemical probes available to study each lipid modification. Protein structures were generated from Protein Databank (http://www.rcsb.org) structures 3N1R (Shh), 5P21 (H-Ras) and 1FMK (c-Src) using The PyMOL Molecular Graphics System, Version 1.3 Schrödinger, LLC.)

The identification and quantification of protein lipidation is a non-trivial challenge, particularly owing to the lack of any intrinsic handle enabling efficient visualisation or isolation of the lipid-modified proteome. Traditional means to study of protein lipidation utilises radiolabelled lipids for metabolic labelling coupled to autoradiography. These procedures are laborious, hazardous and require weeks to months of exposure time to obtain results. The development of chemical proteomics [11] in recent 
years has revolutionised the study of PTMs, in particular protein lipidation [1, 11, 12]. New chemical tools and techniques now allow the facile and rapid detection, identification and quantification of protein lipidation in a matter of days $[11,12]$.

In this short account we cover the basic principles of chemical proteomics in the study of protein acylation, prenylation and cholesterylation, as well as recent advances in quantitative methods to study these PTMs.

\section{Introducing a Chemical Handle on Lipidated Proteins}

The most widely applicable chemical method for labelling and isolating lipidated proteins involves metabolic labelling in cultured cells or enzymatic labelling in vitro with an alkyne or azide-tagged lipid analogue [11-19]. The small, biologically inert chemical tag is minimally disruptive allowing recognition and transfer onto target proteins by cellular lipid transferase enzymes. The alkyne/azide chemical tag provides a handle for down-stream processing, enabling further labelling of the lipidated protein. Tagged proteins can be ligated in vitro to secondary reporter molecules and/or affinity handles in highly specific chemical transformations such as the Huisgen cycloaddition or Staudinger ligation $[11,12,16]$. The proteins can subsequently be visualised by gel based methods or enriched by affinity purification. The enriched, lipidated proteome can be analysed by state of the art massspectrometry (LC-MS/MS). By this method, in excess of 400 lipidated proteins can be identified in a single experiment $[20,21]$.

Metabolic labelling with lipid analogues is applicable to each of the lipid PTMs discussed above. In addition, several other methods such as acyl-biotin exchange (ABE) and resin assisted capture (acylRAC) have been developed for the study of protein palmitoylation and will be further detailed in following sections.

(FIGURE 2. Metabolic tagging of lipidated proteins. Chemically tagged lipid probes are transferred to target proteins in cell culture by lipid transferase enzymes. After cell lysis the metabolically tagged proteins can be further functionalised by addition of secondary reporter molecules of choice such as biotin and/or flourophores. The flourophore enables rapid detection of lipidated proteins by in-gel fluorescence. The biotin affinity handle allows purification of lipidated proteins and further analysis and quantification by Western blots of proteomics.)

\section{Protein Acylation: Myristoylation and Palmitoylation}

Myristoylation and palmitoylation are the attachment of a myristate (C14) or palmitate (C16) fatty acid chain respectively. Myristoylation, catalysed by $N$-myristoyl transferase (NMT1 and NMT2 in humans), occurs on an N-terminal glycine, either co- or post-translationally, forming an irreversible amide [1]. Palmitoylation, on the other hand, is a reversible, dynamic process controlled by both palmitoyl acyl transferase (PAT) and palmitoyl thioesterase enzymes [22]. The palmitate moiety is attached to one or more internal cysteine residues via a thio-ester bond. In some instances $N$ palmitoylation, resulting in an amide bond, results from the rearrangement from an $S$-palmitoylated residue [2].

Our group has pioneered the use of chemical tools to study protein $N$-myristoylation through metabolic labelling using azido and alkynyl tagged myristic acid analogues [15-17]. The myristic acid analogues are recognised by acyl-CoA synthase and converted to the corresponding CoA-thioesters, which then functions as a substrate for NMT [16]. The chemical tag introduced on myristoylated proteins enables visualisation of the myristome by gel-based methods, as well as enrichment for immunoblotting or proteomic analysis [23, 24].

Recent studies have focused on methods to identify and quantify dynamic protein palmitoylation. Similarly to myristoylation, palmitoylated proteins can be detected by means of azido or alkynederivitised palmitoyl analogues $[14,19,25]$. Cravatt and Martin along with other groups in the field pioneered the use of 17-octadecynoic acid (17-ODYA) for metabolic labelling of palmitoylated proteins [26]. 
The labile nature of the $S$-palmitoyl-cysteine thioester bond presents challenges but also advantages in the detection of palmitoylated proteins that are not encountered for other forms of protein lipidation. A number of analytical techniques including ABE [27-29], acyl-RAC [30] and isotope-coded fatty acid transmethylation (iFAT) [31] exploit the labile nature of the palmitate-cysteine thioester bond, and involve chemical transformation of the native proteome only after cell or tissue lysis. In ABE and acyl-RAC free thiols are capped, followed by cleavage of the palmitoyl thioester bond by hydroxylamine to expose a free sulfhydryl group. In ABE the free thiol is coupled to a biotin group, allowing enrichment on streptavidin resin. In the acyl-RAC protocol, the protein sample is instead incubated with a thiol-reactive resin, allowing capture and enrichment to proceed in one step. Although powerful, relying solely on thiol and thioester chemistry to label targets results in relatively high background (particularly in more complex systems) and loss of any information on the identity of the species (lipid or otherwise) previously bound via a thioester.

In iFAT proteins are separated by SDS-PAGE, stained and each band excised before subsequent processing. Each band is subjected to base-catalysed transmethylation with isotopically labelled methanol to generate the fatty acid ester, which is subsequently extracted and identified by mass spectrometry. In contrast to $\mathrm{ABE}$ and acyl-RAC which aim to identify the palmitoylated protein and cannot give any information about the fatty acid modification, iFAT allows the identification and quantification of the modifying fatty acid [31]. Further elaboration of the iFAT protocol, such as immuno-purification before transmethylation, would be required to allow assignment of a particular lipid to a specific protein.

$\mathrm{ABE}$ has been widely utilised to study protein palmitoylation in a range of biological systems, e.g. yeast [28], murine [29], human [32, 33] and African trypanosomes [34]. A recent report from the Rayner group [21] presented a comprehensive study of protein palmitoylation in Plasmodium falciparum. Analysis of late stage asexual parasites cultured in human erythrocytes using a combination of $\mathrm{ABE}$ and metabolic labelling with 17-ODYA enabled the detection of more than 400 palmitoylated proteins. This report presents an interesting side-by-side comparison of the two most commonly used techniques for detecting protein palmitoylation. Each technique detected a unique pool of proteins, with an overlap of ca. 50\%. More proteins were detected by ABE, but this technique was also associated with higher background.

(TABLE 1. Summary of key advantages/disadvantages and potential applications of metabolic tagging, ABE/acyl-RAC and iFAT.)

\section{Prenylation and Cholesterylation}

Protein prenylation, the attachment of a farnesyl (C15) or geranylgeranyl (C20) isoprenoid to one or more cysteine residues located near protein $\mathrm{C}$-terminus are post-translational modifications that modulate protein cellular localisation, signalling and degradation [3]. The transfer is catalysed by a dedicated transferase enzyme, farnesyl transferase (FTase) or geranylgeranyl transferase type I or II (GGTase-1 or GGTase-2/RabGGTase) from a pyrophosphate prenyl donor. The prenyl tail allows proteins to localise to the cell membrane where they can exert their function and interact with downstream effectors. Many key members of the Ras superfamily of proteins, including Rho and Rab GTPases require prenylation for correct function. As several cancers are associated with dysfunctional Ras signalling, prenyl transferase inhibitors have received much attention as potential anti-cancer treatments $[10,35]$.

Several labs [18, 24, 36, 37], including our own [13], have reported the use of chemical reporters to detect and identify prenylated proteins. Two different strategies are generally employed: 1) metabolic labelling using prenyl alcohols in cell culture or 2) in vitro prenylation with prenyl pyrophosphates using recombinant transferases in cell lysate or tissue homogenate. The prenyl alcohols are taken up by cells in culture and converted to their pyrophosphate equivalent through a poorly characterised isoprenoid salvage pathway [38]. For adequate incorporation of prenyl probes pre- and/or co- 
treatment with statins is commonly required. Farnesyl- and geranylgeranyl pyrophosphate are intermediates of cholesterol biosynthesis, and hence statin treatment leads to depletion of endogenous isoprenoids by inhibiting the rate-limiting enzyme of the mevalonate pathway, HMG-CoA reductase. Labelling of the prenylated proteome is hence limited by the activity of the prenyl alcohol salvage pathway and competition with pools of endogenous isoprenoids. Statin treatment, however, has multiple effects in the cell including altered expression of several prenylated proteins [39].

The differential detection of farnesylated versus geranylgeranylated proteins has not yet been quantitatively accessed with currently available chemical tools. Reported probes are either nonselective or suitable for specific detection of geranylgeranylation. Several studies suggest that the prenylation state of e.g. RhoB, a protein that can be either fanesylated or geranylgeranylated, promotes different functions in the cell and affects cellular localisation [9]. Furthermore, it has been suggested that proteins normally farnesylated, e.g. Ras proteins, are geranylgeranylated in the presence of farnesyl transferase inhibitors (FTIs), and this may in part explain the failure of FTIs to translate from promising anti-cancer drugs in cell studies to useful agents in the clinic $[10,35]$.

Protein cholesterylation is a post-translational modification required for the function and signalling of Hedgehog (Hh) proteins [4]. Misregulated Hh signalling is implicated in a number of cancers, including pancreatic carcinoma and lung adenocarcinoma. Our group recently reported the first chemically tagged probe for probing protein cholesterylation in live cells [14]. This probe labels Sonic Hh (Shh) proteins transiently over-expressed in HEK 293a cells and carcinoma cell lines. In-gel fluorescence analysis of the cholesterylated proteome also indicated that a number of other proteins may be labelled by the cholesterol probe, but their identity has yet to be established. Furthermore, the same paper reported the first example of tagging both $\mathrm{Shh} N$-palmitoylation and $O$-cholesterylation in the same cell by metabolic labelling with a combination of mutually compatible palmitate and cholesterol probes.

\section{Quantification of Protein Lipidation}

A steady stream of reports published in the past few years has presented novel methodologies and their application to identify and quantify lipidated proteins. This development has been greatly facilitated by concurrent advances in sophisticated mass spectrometry, and its increasingly widespread availability. Current technology has sufficient resolution power to identify and quantify in excess of 10,000 proteins from complex mixtures such as cell or tissue lysates [40].

In recent years quantitative proteomic techniques such as stable isotope labelling with amino acids in cell culture (SILAC) [41] have been utilised in combination with chemical proteomics to quantify protein lipidation. The combination of these techniques presents exciting opportunities to understand changes in protein lipidation in response to disease, drugs, treatment, as well as changes in other metabolic and signalling pathways in the cell. Furthermore, quantification can be used as a means to assess signal-to-background ratios and validate proteomics hits [20,21]. Although these technologies can be costly to implement, the increased value of the data in terms of systems modelling will result in increasingly widespread use SILAC or other quantitative approaches in combination with chemical tagging. A useful variation on this theme is spike-in SILAC [42], recently developed by the Mann group, which may find application in the quantification of protein lipidation.

In cases where the use of SILAC is impractical or not feasible, such as in vivo or in primary cell culture where the stability of the cell phenotype is limited to a few passages, other quantitative methods such as isobaric tagging (e.g. iTRAQ) or ICAT can prove useful. For example, ICAT has been applied to survey the targets of palmitoyl acyltransferase DHHC2 [43].

\section{Challenges in the Field and Future Perspectives}

Whereas great advances have been made in the study of protein lipidation in vitro and in cell culture only a few groups have reported the use of chemical probes to study in vivo systems, for example by metabolic labelling with $\omega$-alkynyl-palmitate [44] or in vitro prenylation of tissue lysates [13]. 
Metabolic labelling in mammals has not yet provided comprehensive or reliable profiling of targets, and further studies will be required to understand whether attendant pharmacokinetic issues (bioavailability/distribution, metabolism, toxicity) can be overcome. However, it seems plausible that certain compartments of an in vivo model would be relatively accessible to metabolic labelling probes, at least over a limited timeframe, for example by direct introduction into the bloodstream or injection into specific organ or xenograft tissue. Although limited to analysis of $S$-acylation, protocols like ABE/acyl-RAC may be more straightforwardly applied to in vivo studies as all down-stream processing steps are done in vitro.

To date there has been little focus on studying the metabolism of the lipid analogues used in labelling studies, and the assumption is commonly made that the proteins identified are modified by the probes used for labelling, and not their metabolites. A recent report suggests that metabolism of analogues does indeed occur, as one would expect for a biomimetic substrate, and that metabolism may also be monitored using the chemical tag [45]. Here, tools to identify both the site of modification and the modifying lipid species would be hugely beneficial, for example using capture-release reagents to aid recovery and analysis of the modified peptide that is otherwise lost in the enrichment step [46].

The field of chemical proteomics is revolutionising the study of protein lipidation. Although great advances have been made, there exist many exciting opportunities to develop new tools and better analytical methodologies. Equally, efforts are ongoing to expand application of the tools already at hand to broaden our understanding of the function and regulation of protein lipidation, and the potential to target it for therapeutic benefit.

\section{Funding}

E.M.S. is funded by a PhD studentship from the British Heart Foundation. R.A.S is funded by a Marie Curie Intra-European Research Fellowship.

\section{References}

[1] Wright, M.H, Heal, W.P., Mann D.J. and Tate E.W. (2010) Protein myristoylation in health and disease. J. Chem. Biol. 3, 19-35

[2] Salaun, C., Greaves, J. and Chamberlain, L.H. (2010) The intracellular dynamic of protein palmitoylation. J. Cell Biol. 191, 1229-1238

[3] Zhang, F.L. and Casey, P.J. (1996) Protein prenylation: molecular mechanisms and functional consequences. Annu. Rev. Biochem. 65, 241-269

[4] Porter, J.A., Young, K.E. and Beachy, P.A. (1996) Cholesterol modification of hedgehog signaling proteins in animal development. Science 274, 255-259

[5] Maeda, Y. and Kinoshita, T. (2011) Structural remodeling, trafficking and functions of glycosylphosphatidylinositol-anchored proteins. Prog. Lipid Res. 50, 411-424

[6] Yu, Z., Brannigan, J.A., Moss, D.K., Brzozowski, A.M., Wilkinson, A.J., Holder, A.A., Tate, E.W. and Leatherbarrow, R.J. (2012) Design and synthesis of inhibitors of Plasmodium falciparum $N$ myristoyltransferase, a promising target for antimalarial drug discovery. J. Med. Chem. doi: $10.1021 / \mathrm{jm} 301160 \mathrm{~h}$

[7] Goncalves, V., Brannigan, J.A., Whalley, D., Ansell, K.H., Saxty, B., Holder, A.A., Wilkinson, A.J., Tate, E.W. and Leatherbarrow, R.J. (2012) Discovery of Plasmodium vivax $N$ myristoyltransferase inhibitors: screening, synthesis, and structural characterization of their binding mode. J. Med. Chem. 55, 3578-3582 
[8] Stigter, E.A., Guo, Z., Bon, R.S., Wu, Y.W., Choidas, A., Wolf, A., Menninger, S., Waldmann, H., Blankenfeldt, W. and Goody, R.S. (2012) Development of selective, potent RabGGTase inhibitors. J. Med. Chem. 55, 8330-40

[9] Storck, E.M. and Wojciak-Stothard, B. (2012) Rho GTPases in pulmonary vascular dysfunction. Vasc. Pharmacol. doi: 10.1016/j.vph.2012.09.004

[10] Triola, G., Waldmann, H. and Hedberg, C. (2012) Chemical biology of lipidated proteins. ACS Chem. Biol. 7, 87-99

[11] Tate, E.W. (2008) Recent advances in chemical proteomics: exploring the post-translational proteome. J. Chem. Biol. 1, 17-26

[12] Heal, W.P. and Tate, E.W., (2010) Getting a chemical handle on protein post-translational modification. Org. Biomol. Chem. 8, 731-738

[13] Berry, A.F., Heal, W.P., Tarafder, A.K., Tolmachova, T., Baron, R.A., Seabra, M.C. and Tate, E.W. (2010) Rapid multilabel detection of geranylgeranylated proteins by using bioorthogonal ligation chemistry. Chembiochem 11, 771-773

[14] Heal, W.P., Jovanovic, B., Bessin, S., Wright, M.H., Magee, A.I. and Tate, E.W. (2011) Bioorthogonal chemical tagging of protein cholesterylation in living cells. Chem. Commun. 47, 40814083

[15] Heal, W.P., Wickramasinghe, S.R., Bowyer, P.W., Holder, A.A., Smith, D.F., Leatherbarrow, R.J. and Tate, E.W. (2008) Site-specific N-terminal labelling of proteins in vitro and in vivo using $N$ myristoyl transferase and bioorthogonal ligation chemistry. Chem. Commun. 28, 480-482

[16] Heal W.P., Wickramasinghe S.R., Leatherbarrow R.J. and Tate E.W. (2008) N-Myristoyl transferase-mediated protein labelling in vivo. Org. Biomol. Chem. 6, 2308-2315

[17] Heal W.P., Wright M.H., Thinon E. and Tate E.W. (2012) Multifunctional protein labeling via enzymatic N-terminal tagging and elaboration by click chemistry. Nat. Protoc. 7, 105-117

[18] Kho, Y., Kim, S.C., Jiang, C., Barma, D., Kwon, S.W., Cheng, J., Jaunbergs, J., Weinbaum, C., Tamanoi, F., Falck, J. and Zhao, Y. (2004) A tagging-via-substrate technology for detection and proteomics of farnesylated proteins. Proc. Natl. Acad. Sci. USA 101, 12479-12484

[19] Hang, H.C., Geutjes, E.J., Grotenbreg, G., Pollington, A.M., Bijlmakers, M.J. and Ploegh, H.L. (2007) Chemical probes for the rapid detection of fatty-acylated proteins in mammalian cells. J. Am. Chem. Soc. 129, 2744-2745

[20] Martin, B.R., Wang, C., Adibekian, A., Tully, S.E. and Cravatt, B.F. (2012) Global profiling of dynamic protein palmitoylation. Nat. Methods 9, 84-89

[21] Jones, M.L., Collins, M.O., Goulding, D., Choudhary, J.S. and Rayner, J.C. (2012) Analysis of protein palmitoylation reveals a pervasive role in Plasmodium development and pathogenesis. Cell Host Microbe 12, 246-258

[22] Bijlmakers, M.J. and Marsh, M. (2003) The on-off story of protein palmitoylation. Trends Cell Biol. 13, 32-42

[23] Wilson, J.P., Raghavan, A.S., Yang, Y.Y., Charron, G. and Hang, H.C. (2011) Proteomic analysis of fatty-acylated proteins in mammalian cells with chemical reporters reveals $S$-acylation of histone H3 variants. Mol. Cell. Proteomics 10, M110.001198 
[24] Liu, K., Yang, P.Y., Na, Z. and Yao, S.Q. (2011) Dynamic monitoring of newly synthesized proteomes: up-regulation of myristoylated protein kinase A during butyric acid induced apoptosis. Angew. Chem. Int. Ed. 50, 6776-6781

[25] Kostiuk, M.A., Corvi, M.M., Keller, B.O., Plummer, G., Prescher, J.A., Hangauer, M.J., Bertozzi, C.R., Rajaiah, G., Falck, J.R. and Berthiaume, L.G. (2008) Identification of palmitoylated mitochondrial proteins using a bio-orthogonal azido-palmitate analogue. FASEB J. 22, 721-732

[26] Martin, B.R. and Cravatt, B.F. (2009) Large-scale profiling of protein palmitoylation in mammalian cells. Nat. Methods 6, 135-138

[27] Drisdel, R.C. and Green, W.N. (2004) Labeling and quantifying sites of protein palmitoylation. Biotechniques 36, 276-285

[28] Roth, A.F., Wan, J., Bailey, A.O., Sun, B., Kuchar, J.A., Green, W.N., Phinney, B.S., Yates, J,R., $3^{\text {rd }}$ and Davis, N.G. (2006) Global analysis of protein palmitoylation in yeast. Cell 125, 1003-1013

[29] Wan, J., Roth, A.F., Bailey, A.O. and Davis, N.G. (2007) Palmitoylated proteins: purification and identification. Nat. Protoc. 2, 1573-1584

[30] Forrester, M.T., Hess, D.T., Thompson, J.W., Hultman, R., Moseley, M.A., Stamler, J.S and Casey, P.J. (2011) Site-specific analysis of protein S-acylation by resin-assisted capture. J. Lipid Res. 52, 393-398

[31] Dong, L., Li, J., Li, L., Li, T. and Zhong, H. (2011) Comparative analysis of S-fatty acylation of gel-separated proteins by stable isotope-coded fatty acid transmethylation and mass spectrometry.

Nat. Protoc. 6, 1377-1390

[32] Dowal, L., Yang, W., Freeman, M.R., Steen, H. and Flaumenhaft, R. (2011) Proteomic analysis of palmitoylated platelet proteins. Blood 118, e62-73

[33] Marin, E.P., Derakhshan, B., Lam, T.T., Davalos, A. and Sessa, W.C. (2012) Endothelial cell palmitoylproteomic identifies novel lipid-modified targets and potential substrates for protein acyl transferases. Circ. Res. 110, 1336-1344

[34] Emmer, B.T., Nakayasu, E.S., Souther, C., Choi, H., Sobreira, T.J., Epting, C.L., Nesvizhskii, A.I., Almeida, I.C. and Engman, D.M. (2011) Global analysis of protein palmitoylation in African trypanosomes. Eukaryot. Cell 10, 455-63

[35] Berndt, N., Hamilton, A.D. and Sebti, S.M. (2011) Targeting protein prenylation for cancer therapy Nat. Rev. Cancer 11, 775-791

[36] Chan, L.N., Hart, C., Guo, L., Nyberg, T., Davies, B.S., Fong, L.G., Young, S.G., Agnew, B.J. and Tamanoi, F. (2009) A novel approach to tag and identify geranylgeranylated proteins. Electrophoresis 30, 3598-3606

[37] Nguyen, U.T., Guo, Z., Delon, C., Wu, Y., Deraeve, C., Fränzel, B., Bon, R.S., Blankenfeldt, W., Goody, R.S., Waldmann, H., Wolters, D. and Alexandrov, K. (2009) Analysis of the eukaryotic prenylome by isoprenoid affinity tagging. Nat. Chem. Biol. 5, 227-235

[38] Bentinger, M., Grünler, J., Peterson, E., Swiezewska, E. and Dallner, G. (1998) Phosphorylation of farnesol in rat liver microsomes: properties of farnesol kinase and farnesyl phosphate kinase. Arch. Biochem. Biophys. 353, 191-198 
[39] Turner, S.J., Zhuang, S., Zhang, T., Boss, G.R. and Pilz, R.B. (2008) Effects of lovastatin on Rho isoform expression, activity, and association with guanine nucleotide dissociation inhibitors.

Biochem. Pharmacol. 75, 405-13

[40] Geiger, T., Wehner, A., Schaab, C., Cox, J. and Mann, M. (2012) Comparative proteomic analysis of eleven common cell lines reveals ubiquitous but varying expression of most proteins. Mol. Cell. Proteomics. 11, M111.014050

[41] Ong, S.E., Blagoev, B., Kratchmarova, I., Kristensen, D.B., Steen, H., Pandey, A. and Mann, M. (2002) Stable isotope labeling by amino acids in cell culture, SILAC, as a simple and accurate approach to expression proteomics. Mol. Cell. Proteomics 1, 376-386.

[42] Geiger, T., Wisniewski, J.R., Cox, J., Zanivan, S., Kruger, M., Ishihama, Y. and Mann, M. (2011) Use of stable isotope labeling by amino acids in cell culture as a spike-in standard in quantitative proteomics. Nat. Protoc. 6, 147-157

[43] Zhang, J., Planey, S.L., Ceballos, C., Stevens, S.M., Jr, Keay, S.K. and Zacharias, D.A. (2008) Identification of CKAP4/p63 as a major substrate of the palmitoyl acyltransferase DHHC2, a putative tumor suppressor, using a novel proteomics method. Mol. Cell. Proteomics 7, 1378-1388

[44] Yap, M. C., Kostiuk, M. A., Martin, D. D., Perinpanayagam, M. A., Hak, P. C., Siddam, A., Majjigapu, J. R., Rajaiah, G., Keller, B. O., Prescher, J. A., Wu, P., Bertozzi, C. R., Falck, J. R. and Berthiaume, L. G. (2010) Rapid and selective detection of fatty acylated proteins using omegaalkynyl-fatty acids and click chemistry. J. Lipid Res. 51, 1566-1580

[45] Thiele, C., Papan, C., Hoelper, D., Kusserow, K., Gaebler, A., Schoene, M., Piotrowitz, K., Lohmann, D., Spandl, J., Stevanovic, A., Shevchenko, A. and Kuerschner, L. (2012) Tracing Fatty Acid Metabolism by Click Chemistry. ACS Chem. Biol. doi: 10.1021/cb300414v

[46] Weerapana, E., Speers, A.E. and Cravatt, B.F. (2007) Tandem orthogonal proteolysis-activitybased protein profiling (TOP-ABPP) - a general method for mapping sites of probe modification in proteomes. Nat. Protoc. 2,1414-1425 


\begin{tabular}{|c|c|c|c|}
\hline Technique & + & - & ? \\
\hline $\begin{array}{l}\text { Metabolic } \\
\text { tagging }\end{array}$ & $\begin{array}{l}\text { - Applicable to all lipid } \\
\text { PTMs } \\
\text { - Amenable to live cell and } \\
\text { in vitro labelling } \\
\text { - Transferase dependent } \\
\text { lipidation }\end{array}$ & $\begin{array}{l}\text { - May require synthesis of } \\
\text { probes } \\
\text { - Competition with } \\
\text { endogenous lipids } \\
\text { - Possible } \\
\text { toxicity/metabolism/bio- } \\
\text { availability issues }\end{array}$ & $\begin{array}{l}\text { - Combine with click-capture } \\
\text { reagents for identification } \\
\text { of modified residue? } \\
\text { - Tracking of lipid } \\
\text { metabolism? }\end{array}$ \\
\hline $\begin{array}{c}\text { ABE/ } \\
\text { Acyl-RAC }\end{array}$ & $\begin{array}{l}\text { - No perturbation of the } \\
\text { biological system } \\
\text { - Commercially available } \\
\text { reagents }\end{array}$ & $\begin{array}{l}\text { - Only applicable to } \\
S \text {-palmitoylation } \\
\text { - High background \& false } \\
\text { positive rate } \\
\text { - No information on } \\
\text { modifying lipid } \\
\text { - Lengthy protocol (ABE) }\end{array}$ & \\
\hline iFAT & $\begin{array}{l}\text { - No perturbation of the } \\
\text { biological system } \\
\text { - ID of modifying lipid } \\
\text { - Commercially available } \\
\text { reagents }\end{array}$ & $\begin{array}{l}\text { - Only applicable to } \\
S \text {-palmitoylation } \\
\text { - No information on } \\
\text { modified protein }\end{array}$ & $\begin{array}{l}\text { - Combine with IP for } \\
\text { protein identification? }\end{array}$ \\
\hline
\end{tabular}




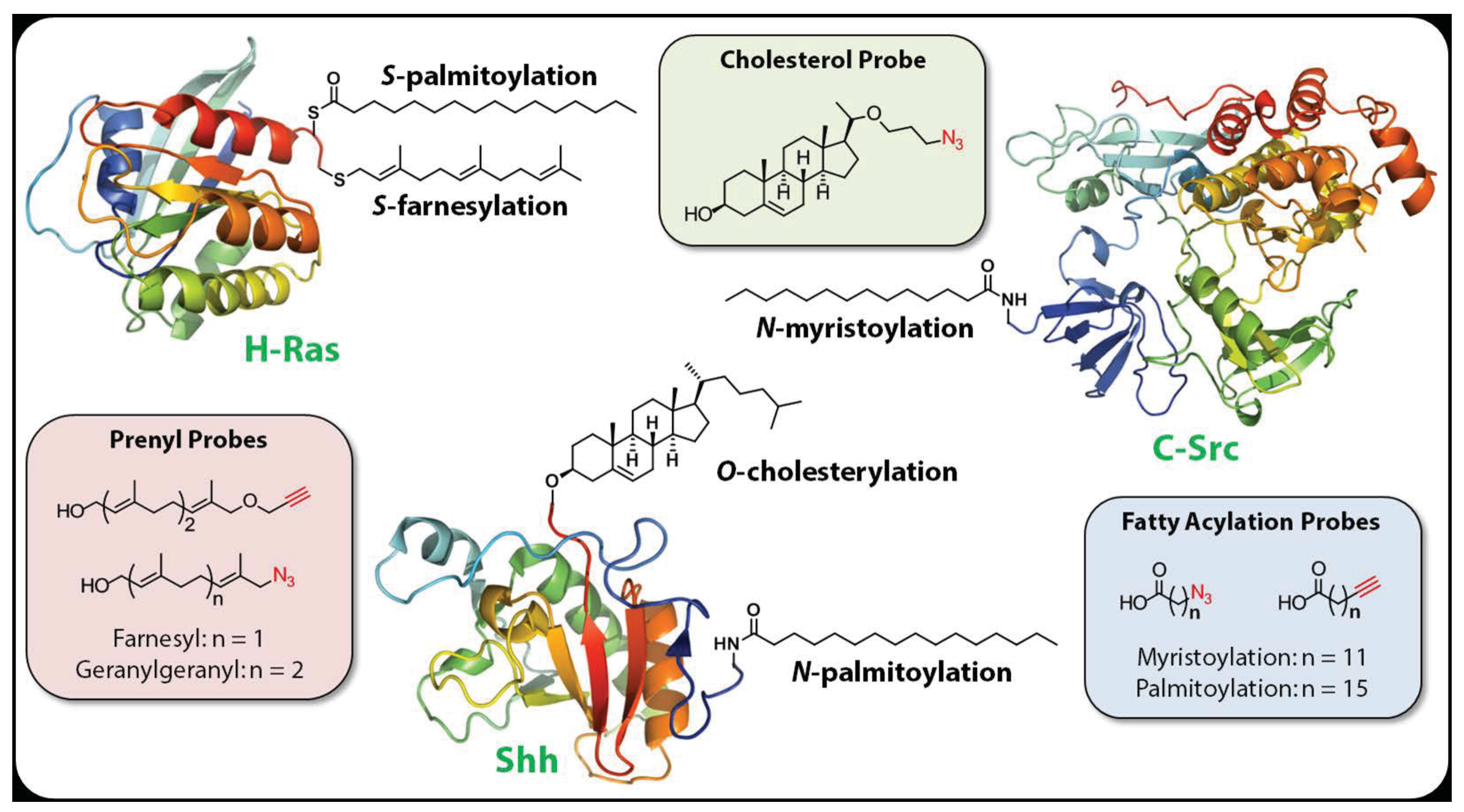




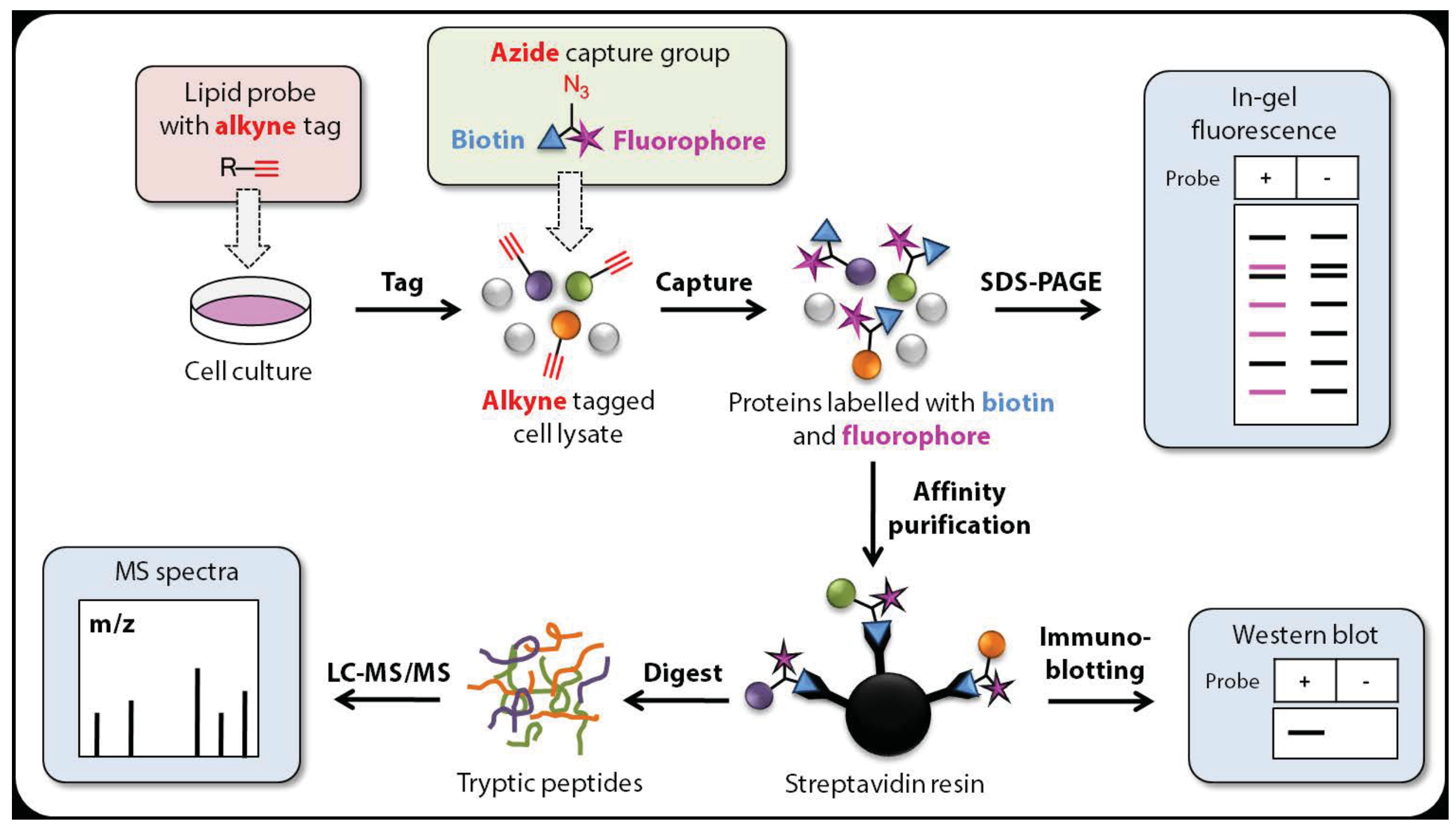




\begin{tabular}{|c|c|c|c|}
\hline Technique & + & - & $?$ \\
\hline $\begin{array}{l}\text { Metabolic } \\
\text { tagging }\end{array}$ & $\begin{array}{l}\text { - Applicable to all lipid PTMs } \\
\text { - Amenable to live cell and in vitro } \\
\text { labelling } \\
\text { - Transferase dependent lipidation }\end{array}$ & $\begin{array}{l}\text { - May require synthesis of probes } \\
\text { - Competition with endogenous lipids } \\
\text { - Possible toxicity/metabolism/ } \\
\text { bio-availability issues }\end{array}$ & $\begin{array}{l}\text { - Combine with click-capture reagents } \\
\text { for identification of modified residue? } \\
\text {-Tracking of lipid metabolism? }\end{array}$ \\
\hline $\begin{array}{l}\text { ABE/ } \\
\text { Acyl-RAC }\end{array}$ & $\begin{array}{l}\text { - No perturbation of the biological } \\
\text { system } \\
\text { - Commercially available reagents }\end{array}$ & $\begin{array}{l}\text { - Only applicable to S-palmitoylation } \\
\text { - High background \& false positive rate } \\
\text { - No information on modifying lipid } \\
\text { - Lengthy protocol (ABE) }\end{array}$ & \\
\hline iFAT & $\begin{array}{l}\text { - No perturbation of the biological } \\
\text { system } \\
\text { - ID of modifying lipid } \\
\text { - Commercially available reagents }\end{array}$ & $\begin{array}{l}\text { - Only applicable to S-palmitoylation } \\
\text { - No information on modified protein }\end{array}$ & $\begin{array}{l}\text { - Combine with IP for protein } \\
\text { identification? }\end{array}$ \\
\hline
\end{tabular}

